\title{
Métodos de la educación musical para el desarrollo de la memoria musical de los estudiantes de música
}

\section{Methods of musical education for the development of the musical memory of music students}

\author{
Benjamín Velazco Reyes ${ }^{1, a}$ \\ https://orcid.org/0000-0003-2780-786X
}

\author{
Wilber Cesar Calsina Ponce ${ }^{1, b}$ \\ https://orcid.org/0000-0001-9094-7684
}

Renzo Favianni Valdivia Terrazas ${ }^{1, \mathrm{c}}$

https://orcid.org/0000-0001-8222-3264

\section{David Ruelas Vargas ${ }^{2, d}$ \\ https://orcid.org/0000-0003-1315-5493}

${ }^{1}$ Universidad Nacional del Altiplano, Puno, Perú.

${ }^{2}$ Universidad Nacional Jorge Basadre Grohmann, Tacna, Perú.

a benya sax@hotmail.com

b csarartista@hotmail.com

crenzofavianni@hotmail.com

d $\underline{\text { divadrv@hotmail.com }}$

\section{RESUMEN}

La memoria musical es la habilidad para recordar lo oído en el orden y secuencia apropiada en un tiempo real o diferido, en la educación musical cumple un rol importante, ya que sirve para aprender las diferentes formas de praxis que se desarrollan en el aula, comprenderlas y recordarlas; habilidad que los estudiantes del I semestre no logran desarrollar en su 28 entorno básico, por consiguiente nuestro objetivo es precisar el nivel de retención en la memoria musical de diferentes secuencias rítmicas, entonadas y habladas, según la aplicación de tres métodos: Dalcroze, LenMus, y Relación Sonido Color. El enfoque es cuantitativo, ya que la investigación es cuasi experimental, para ello se tomó a los 41 estudiantes del curso de Lenguaje Musical I y los dividimos en dos grupos (experimental y control). Los resultados obtenidos evidencian que los tres métodos aplicados son eficaces para resolver las diferentes combinaciones de dictado tanto rítmico, melódico y de reconocimiento de las notas en el pentagrama. Concluyéndose en que el grado de retención en la memoria musical de diferentes secuencias rítmicas, entonadas y habladas, según la aplicación de tres métodos es significativo en un nivel de logro esperado.

Palabras clave: Aprendizaje del lenguaje musical, audición, memoria auditiva, memoria musical y pensamiento musical.
Recibido el: 24/11/2019

Aceptado el: 27/03/2020

\begin{abstract}
Musical memory is the ability to remember the ear in the appropriate order and sequence, in a real or deferred time; in music education it plays an important role, since it helps us to learn the different forms of praxis that develop in the classroom, understand and remember them; ability that the students of the I semester fail to develop in their basic environment, therefore our objective is to specify the level of retention in the musical memory of different rhythmic sequences, intoned and spoken, according to the application of three methods: Dalcroze, LenMus, and Color Sound Ratio. The approach (epistemological paradigm) is quantitative, since the research is almost experimental, for this we take the 41 students of the Musical Language I course and divide them into two groups (experimental and control). The results show that the three methods applied are effective in solving the different combinations of rhythmic, melodic dictation and note recognition on the staff, concluding that the degree of retention in the musical memory of different rhythmic sequences, toned and spoken, according to the application of three methods is significant at level expected achievement.
\end{abstract}

Keywords: Hearing, learning musical language, listening memory, musical memory and musical thinking. 


\section{INTRODUCCIÓN}

La memoria es básicamente una función cerebral, el ser humano ha logrado desarrollarla permitiéndole una evolución compleja de su especie (Pino, 2011). Esta función permite al organismo codificar, almacenar y recuperar información (Feldman, 2005), todo ello es producto de las complejas conexiones sinápticas repetitivas entre las neuronas, en el sistema nervioso central del cerebro del ser humano (Córdoba, Albert, y López, 2010); la memoria es un elemento indispensable para el desarrollo del ser humano, por lo tanto, "es la capacidad de recordar hechos pasados en los seres dotados de consciencia y también es la capacidad de repetir previamente lo aprendido" (Real Academia Española, 1995).

La memoria musical es fundamental en la formación musical, permite la individualización de los elementos de la música en el cerebro, logrando una mejor asimilación aportando al desarrollo de una excelente carrera como concertista e intérprete (Velásquez, 2010); los músicos expertos logran experiencias de alto nivel gracias a la práctica y educación adquirida en su campo, ya que ellos utilizan las mismas estrategias de los expertos de otras áreas que requieren memorizar grandes cantidades de información (Noice, Jefferey, Noice, y Chaffin, 2008).

El desarrollo de la competencia auditiva, educación o formación del oído musical a través de la memoria, es un proceso lento pero firme, por lo tanto, exitoso, cuando se trabaja simultáneamente la lectura, escritura, audición y ejecución musical a través del canto o de la interpretación de un instrumento musical. La aplicación de un software produce un desarrollo en el pensamiento musical manifestado por la toma de consciencia en las imágenes sonoras o auditivas, cuando el sonido físico no está presente (Martinez, 2008).

Riera (2011) en su investigación realizó la integración de la música y la pintura a través del sonido y el color, como también los diferentes aspectos vinculados a la percepción, a los procesos cognitivos para la interpretación, creación y apreciación del arte como una experiencia cinestésica, donde se establece la correspondencia entre las notas musicales y los colores para crear un nexo entre la escala musical y la escala de color con el fin de representar una melodía de una pieza de música clásica de manera que se identifique los tonos musicales y el ritmo en la obra pictórica.
Por otro lado, Sandoval (2015) como producto de su investigación, manifiesta que la música presenta un efecto transformador sobre el desarrollo de la memoria operativa en niños del nivel de transición 2, sobre todo en cuanto a su carácter fonológico, promoviendo cambios significativos en los niños. Asimismo, Sanches, Flores y Aravena (2003) manifiestan que los niños con discapacidad visual logran desarrollar su memoria de corto plazo a través del audio, para ello los investigadores presentaron un software "Audiomemorice", cuya finalidad es que el sonido constituya un poderoso interfaz para estimular el desarrollo de la memoria de los niños ciegos.

En ese entender Herrera y Cremades (2012) en los resultados de su invetigación muestran que el modelo más adecuado para desarrollar el trabajo de la memoria es la combinación de la memoria visual, auditiva, kinestésica y analítica, asi tambien como las actividades de carácter ludico, motivador y creatico (Berrón, Balsera, y Monreal, 2016).

Elplanteamiento del presente trabajo se fundamenta en que los ingresantes al programa de Música de la Universidad Nacional del Altiplano presentan un nivel pre básico en su formación audio perceptivo (Lenguaje Musical) ya que solo un $10 \%$ de los ingresantes cumplen con los requisitos mínimos para iniciar su carrera profesional, estos datos se evidencian en los resultados finales del curso logrando aprobar solo el 30\%, (encuesta aplicada a los estudiante ingresantes 2018 II), en relación a ello se plantea el objetivo de precisar el nivel de retención en la memoria musical de diferentes secuencias rítmicas, entonadas y habladas, según la aplicación de tres métodos: Dalcroze, LenMus, y Relación Sonido Color.

\section{MARCO TEÓRICO}

\section{Memoria Musical}

La memoria musical es una capacidad especial para conservary recordar una serie de sonidos musicales, cuando se nos presentan, como una melodía o una progresión armónica (Shinn, 1984) que se manifiesta en diferentes características: potencia, facilidad, tenacidad, volubilidad y ordenamiento, se clasifican en tres grados: memorias musicales rápidas para retener, pero que olvidan fácilmente lo que él denomina "Escribir en arena". Memorias musicales que requieren mayor esfuerzo, pero se conservan fuertemente, lo que él denomina 
"Grabado en piedra". Memorias musicales inestables, que presentan datos no requeridos y los empujan a la expresión inoportunamente o tienen contenidos desordenados, con un aporte lento cuando la oportunidad de usarlos ya ha pasado, lo que él llama "Veletas al viento" (Barbacci, 1965).

La música compromete a nuestro cerebro en su totalidad (Clynes, 1982), todos los humanos tienen una capacidad innata para procesar la música (Ibarra, 2009), donde el hemisferio derecho coordina la percepción y memoria musical, el cual aumenta el flujo sanguíneo cerebral en el lóbulo temporal derecho que es el que participa en la audición (Bowers, 2003), es así que la música abarca muchas áreas distribuidas a lo largo del cerebro, incluyendo aquellas que normalmente están involucradas en otro tipo de conocimientos (Weinberg, 2006), así podemos manifestar que la percepción musical surge de la interacción de actividad en ambos lados del cerebro (Cromie, 1997).

Chaffin y Imreh (2002), en un estudio a un painista experto, encontraron que la memoria auditiva y muscular asociadas a la memoria conceptual, al momento de juntarse las gráficas auditivas y musculares, crean una automatización en los movimientos durante la ejecución, mientras la memoria conceptual se utiliza para ubicarse, es decir saber que parte de la obra se está ejecutando. Por otro lado, Chaffin y Logan (2006), afirman que, si existe una organización jerárquica de la memoria, ya que los expertos logran utilizar un mapa mental de la obra, asi podemos manifestar que de todos los dones con que pueden estar dotados los individuos ninguno surge más temprano que el talento musical (Gardner, 1994).

Janata y Grafton(2003)manifiestanque, las regiones del cerebro humano responsables de percibir la música son: a) corteza prefrontal rostromedial, recuerda y procesa los tonos - responsable del aprendizaje de las estructuras musicales. b) lóbulo temporal derecho procesamiento básico del sonido -separa la armonía musical de otros estímulos auditivos. c) sistema límbico, responsable de percibir las emociones - mantiene comunicación con el lóbulo temporal y por ello la música impacta en los sentimientos.

La música es hecha de un gran número de fragmentos pequeños encadenados y la percepción de esta es simplemente la concatenación de una serie de actos perceptuales sobre tales fragmentos (Sloboda, 1985) de donde deriva la inteligencia auditiva, en ese sentido para Willems (2001), la inteligencia auditiva puede ser entendida como una síntesis abstracta de las experiencias sensoriales y afectivas, esto se traduce en comprender la música. Afirma también que, la lectura y escritura musical son medios intelectuales para fijar y transmitir el pensamiento musical sonoro, donde la inteligencia musical se configura a través de la memoria, audición interior, imaginación creadora, acordes, audición relativa y absoluta.

Para ser un buen músico se debe desarrollar una buena escucha, lo que implica haber desarrollado la competencia auditiva para reconocerla por medio de la memoria; para los estudiantes de música es fundamental en su formación profesional el entrenamiento del oído, porque al escuchar cualquier melodía pueda llegar a transcribirla, considerando que transcribir es codificar los signos del lenguaje musical, este último es lo que hace un compositor cuando crea la música en su mente y luego la escribe, donde el papel del intérprete es tocar o cantar dicha música para que el oyente la disfrute, es decir es el medio de comunicación entre el compositor y el auditorio (Alvarado, 2013). La competencia auditiva se desarrolla por medio del calentamiento auditivo que es adiestrar al oído en los aspectos básicos dictado la tonalidad, el compás, los intervalos, el ritmo, la melodía y la armonía, el sonido inicial, el tipo de comienzo, el número de compases que tiene el dictado (Martinez, 2008).

Desde una perspectiva lingüista, "oír" es un fenómeno biológico, que se asocia a la capacidad de distinguir sonidos; "escuchar" pertenece al dominio del lenguaje donde se constituye las interacciones sociales con los demás; la diferencia es que cuando escuchamos entramos al mundo interpretativo, lo que implica compresión (Echeverría, 2002).

Según Paney (2007), la audición o entrenamiento auditivo es una asociación directa entre el oído y el cerebro en el sentido de la capacidad de entender la música desde una perspectiva auditiva, esta se basa en Hedges para afirmar que la teoría de la escritura envuelve la codificación y decodificación de la música conceptualmente, mientras que la teoría de la audición envuelve la codificación y decodificación de la música perceptualmente. 


\section{Método Dalcroze}

El método Dalcroze está basado en la idea de que el alumno debe experimentar la música física, mental y espiritualmente, teniendo como metas principales el desarrollodeloídointerno, asícomo elestablecimiento de una relación consciente entre mente y cuerpo para ejercer control durante la actividad musical. El método es aplicable a un espectro de edades que abarca desde los 3-4 años hasta la edad adulta. En el caso específico de los músicos es importante destacar el trabajo auditivo a través de la discriminación de los diferentes parámetros del sonido (altura, duración, intensidad, timbre), así como de las diferentes tonalidades y modos, mediante una metodología propia (Ráez, 2013).

El método Dalcroze divide la formación musical en tres aspectos que están íntimamente relacionados entre sí: Euritmia, (buen ritmo) entra en el cuerpo del alumno para sentir conscientemente las sensaciones musculares de tiempo y energía en sus manifestaciones en el espacio, el cuerpo se convierte en instrumento y ejecuta o transforma en movimiento en algún aspecto de la música. Solfeo Dalcorziano, desarrollo del oído interno en el alumno para escuchar musicalmente y cantar afinadamente. Improvisación, motiva al estudiante a expresar sus ideas musicales propias estimulando los poderes de concentración y la capacidad de escuchar e imaginar creando sentimientos de satisfacción y logro (Jaques, 1999).

\section{Método LemMus}

Phonascus, en latín "el profesor de música", es un software para el aprendizaje del lenguaje musical, que se puede utilizar para mejorar las habilidades para leer partituras, para mejorar el oído musical o, simplemente, para aprender los principios fundamentales del lenguaje y la teoría de la música (Salmerón, 2013).

LenMus es útil para la educación del oído, la entonación y el dictado musical, resultan difíciles la identificación de intervalos, escalas y acordes y es casi imposible de practicar sin un profesor que ejecute el piano. LenMus Phonascus está siempre a disposición para ello y no necesitas un piano. LenMus incluye los siguientes ejercicios: comparación de intervalos, identificación de intervalos, identificación de notas, identificación de acordes, identificación de escalas, identificación de cadencias, identificación de tonalidades.

\section{Método Relación Sonido Color}

Se caracteriza por proporcionar apoyo visual por medio de colores que representan cada uno de los sonidos musicales utilizados, la teoría del sonido y su relación con el color se fundamenta en la experiencia de Isaac Newton del paso de la luz por el prisma y el resultado fue los siete colores del arco iris, del mismo modo se establece una relación a manera de analogía, con los sonidos musicales de la escala. (Cidoncha, 2011).

Los siete sonidos son representados por los siete colores del prisma o del arco iris según la teoría del color de Isaac Newton. (Lopez, et al., 2005).

Tabla 1.

Datos de Notas Musicales y de Tonos de Color Analizados.

\begin{tabular}{lllll}
\hline $\begin{array}{l}\text { Sonido } \\
\lambda(\mathrm{nm})\end{array}$ & Sonido & $\begin{array}{l}\text { Sonido } \\
f(\mathrm{~Hz})\end{array}$ & $\begin{array}{l}\text { Color } \\
\lambda(\mathrm{nm})\end{array}$ & $\begin{array}{l}\text { Color } \\
f(\mathrm{THz})\end{array}$ \\
\hline 16.504 & Eo & 20.601 & 780 & 384 \\
11.670 & Ao\# & 29.134 & 755 & 397 \\
8.252 & E1 & 41.202 & 730 & 411 \\
5.835 & A1\# & 58.268 & 705 & 425 \\
4.126 & E2 & 82.404 & 680 & 441 \\
2.918 & A2\# & 116.537 & 655 & 458 \\
2.063 & E3 & 164.808 & 630 & 476 \\
1.459 & A3\# & 233.074 & 605 & 496 \\
1.032 & E4 & 329.616 & 580 & 517 \\
0.729 & A4\# & 466.147 & 555 & 540 \\
0.516 & E5 & 659.232 & 530 & 566 \\
0.365 & A5\# & 932.295 & 505 & 594 \\
0.258 & E6 & 1318.464 & 480 & 625 \\
0.182 & A6\# & 1864.590 & 455 & 659 \\
0.129 & E7 & 2636.928 & 430 & 697 \\
0.091 & A7\# & 3729.179 & 405 & 740 \\
0.064 & E8 & 5273.856 & 380 & 789 \\
\hline
\end{tabular}

Fuente: Color y Música: Relaciones Físicas entre Tonos de Color y Notas Musicales. (Perez y Gilabert, 2010).

Las letras que representan a los sonidos son $\mathrm{E}$ y A\# y equivalen a Mi y a La\#. Los sufijos van del 0 al 8 e indican la altura de esas notas, 0 sería el valor de menor frecuencia (más grave) y 8 el de mayor frecuencia (más agudo). Cuando los valores van de E0 a E1 la distancia es de una octava (ocho notas desde Mi a Mi) la que apreciamos en la figura 1. 


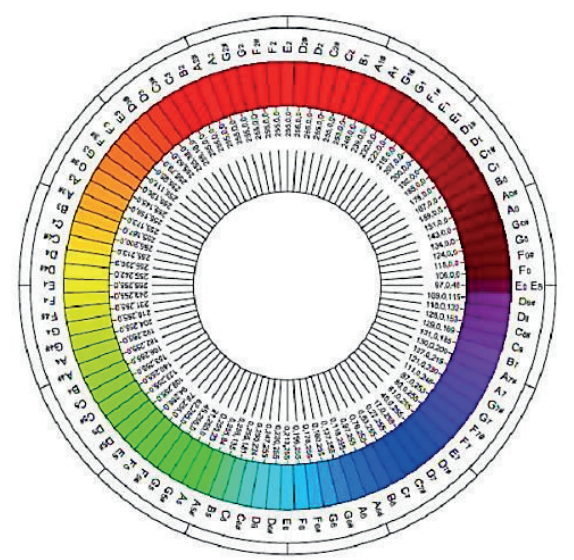

Figura 1. Óptica Pura y Aplicada Fuente: Color y Música: Relaciones Físicas entre Tonos de Color y Notas Musicales. (Perez y Gilabert, 2010).

Según Aschero la numerofonia parte de la relación entre la imagen y el sonido, de la vinculación entre los sentidos más potentes que son la vista y el oído. Este es un código interactivo de las áreas fisicomatemáticas, de origen pitagórico, el cual se ha desarrollado con pensar científico, integrando la óptica, la acústica, la geometría y la aritmética, en un modelo único de representación simbólica, así como apreciamos en la figura 2 (Aschero, 2012).



Figura 2. Numerofonia de los Sonidos en DO Fuente: Numerofonia (Aschero, 2012).

El color indica la altura del sonido, por ejemplo, el rojo es el Sonido DO. La escala utilizada por Aschero tiene doce sonidos al igual que la escala cromática de un piano, de ahí que los colores conforman la escala cromática de color en relación directa con la escala cromática de los sonidos musicales como indica la tabla 2.

Tabla 2.

Relación del Sonido Color Según Aschero

\begin{tabular}{clc}
\hline $\mathrm{N}^{\circ}$ & \multicolumn{1}{c}{ Nota } & Color \\
\hline 12 & SI & Purpura \\
11 & SI bemol o La Sostenido & Magenta \\
10 & LA & Violeta \\
9 & LA bemol o SOL sostenido & Azul \\
8 & SOL & Cobalto \\
7 & SOL bemol o FA sostenido & Cian \\
6 & FA & Esmeralda \\
5 & MI & Verde \\
4 & MI bemol o RE sostenido & Lima \\
3 & RE & Amarillo \\
2 & RE bemol o DO sostenido & Anaranjado \\
1 & DO & Rojo \\
\hline Fuente: & Numerofonia (Aschero, 2012). &
\end{tabular}

\section{MATERIALES Y MÉTODOS}

El paradigma de investigación en el que se enmarca el trabajo es el positivista, porque se basa en procedimientos de análisis de datos como los establecidos en las ciencias exactas, tal como hacen las leyes que explican los fenómenos naturales o físicos (Cohen y Manion, 2003). Dentro de este paradigma queda posicionada la investigación cuantitativa, donde esta utiliza la recopilación de información para poner a prueba o comprobar las hipótesis mediante el uso de estrategias estadísticas basadas en la medición numérica, (Hernández, Fernandez, y Baptista, 2014).

\section{Diseño de investigación}

El diseño que se utilizó en la investigación es cuasiexperimental, ya que los sujetos no se asignan al azar a los grupos ni se emparejan, porque tales grupos ya existían, los cuales se llaman grupos intactos, (Campbell y Stanley, 1996), de dos grupos, (control y experimental), con prueba de entrada (Pretest) y Salida (Postest). Se aplicó el tratamiento al grupo experimental, donde el diseño se representa de la siguiente manera:

Tabla 3.

Representación Del Diseño De Investigación

\begin{tabular}{lccc}
\hline G.E. & Y1 & X & Y2 \\
G.C. & Y1 & - & Y2 \\
\hline
\end{tabular}

\section{DESCRIPCIÓN:}

G.E. $=$ Grupo Experimental

G.C. $=$ Grupo Control

$\mathrm{Y} 1=$ Prueba de entrada (Pretest)

Y2 = Prueba de salida (Postest)

$\mathrm{X}=$ Experimento (métodos aplicados)

\section{Técnica}

Se utilizó la Encuesta, dicha técnica se utiliza ampliamente, porque permite obtener y elaborar datos de modo rápido y eficaz, además de ello sirve para recoger datos de las unidades de análisis (Barraza, 2006) y se clasifican según los que solicitan y aportan la información, es decir, según los sujetos involucrados (Rodriguez, 1996).

\section{Instrumentos}

Los instrumentos de medición fueron la prueba de entrada (Pretest) y salida (Postest), apoyados 
en la observación y el diario de campo. Cerda (1993), manifiesta que los instrumentos permiten tener acceso a la información que se necesita para resolver el problema o comprobar la hipótesis.

\section{Pretest}

Sirvió para recoger la primera información acerca del grado de retención en la memoria musical de los estudiantes y estuvo conformado por 3 partes esenciales del lenguaje musical que son el solfeo Rítmico, Entonado, Hablado. Postest: Sirvió para recoger la información final acerca del grado de retención en la memoria musical de los estudiantes y estuvo conformado por 3 partes esenciales del lenguaje musical que son el solfeo Rítmico, Entonado, Hablado. Para jerarquizar el conocimiento obtenido nos basamos en la siguiente tabla.

Tabla 4.

Nivel de retención en la Memoria

\begin{tabular}{ll}
\hline Categoría & Puntuación \\
\hline Logro Esperado & $18-20$ \\
Logro Previsto & $14-17$ \\
En Proceso & $11-13$ \\
En Inicio & $00-10$ \\
\hline
\end{tabular}

Fuente: Escala de rendimiento académico

Diario de campo: Sirvió para registrar los acontecimientos que se suscitaron en el aula, escuela y comunidad. Especialmente en la práctica pedagógica del maestro investigador.

\section{Población}

La población estuvo constituida por los estudiantes del I semestre del Programa de Música, del curso de Lenguaje Musical, oscilando en edades de 17 a 24 años, siendo un total de 41 estudiantes, donde un $95 \%$ son de sexo masculino y $5 \%$ de sexo femenino, todos admitidos a la UNAP mediante un examen de aptitud vocacional y un examen general de conocimientos para el año académico 2018 II. Cabe mencionar que el 90\% de estudiantes provienen de las provincias de la región de Puno $\mathrm{y}$ un $67 \%$ tuvieron su preparación musical en sus colegios (talleres de banda, estudiantina y coro), desarrollando solo la parte básica de teoría, ejecución instrumental, y algunos aspectos de solfeo o entrenamiento auditivo. Un $77 \%$ no lee música en pentagrama, un $84 \%$ ejecuta su instrumento a oído, y el 74\% de ellos toca de memoria. (Fuente: encuesta realizada a los estudiantes de Lenguaje Musical I año académico 2018 II).

\section{Muestra}

Para nuestro estudio utilizamos las muestras diversas o de máxima variación: estas muestras son utilizadas cuando se busca mostrar distintas perspectivas y representar la complejidad del fenómeno estudiado, obien documentar la diversidad para localizar diferencias y coincidencias, patrones y particularidades. (Hernández et al., 2014). sé conformó de la siguiente manera:

Tabla 5.

Muestra de Estudiantes del Curso de Lenguaje Musical

\begin{tabular}{lll}
\hline Ciclo & Grupo & Número \\
\hline I & Experimental & 20 \\
Total & Control & 21 \\
\hline
\end{tabular}

Fuente: tabla 5

\section{Recolección de datos}

Al grupo experimental, se les explicó los instrumentos para la recolección de los datos y como se deben aplicar en función a la investigación, a su vez dicho grupo recibió un tratamiento experimental de las sesiones de aprendizaje de acuerdo a los métodos propuestos para poder consolidar su lenguaje musical. Para el grupo control se desarrollaron las actividades de aprendizaje del lenguaje musical en forma rutinaria tal como se realizaba en los semestres anteriores.

\section{Formulación de la hipótesis estadística}

- Ho: El promedio de notas obtenidas en el Pretest por los estudiantes del grupo experimental es similar a los obtenidos por el grupo control.

$$
\mathrm{Xe}=\mathrm{Xc}
$$

- Ha: El promedio de notas obtenidas en el Postest por los estudiantes del grupo experimental es diferente a los obtenidos por el grupo control.

$$
\mathrm{Xe}>\mathrm{Xc}
$$

- Determinación del nivel se significancia Se utilizó $\alpha=0,05$, que significa error del 5\% y el grado de significación es el $95 \%$.

- Prueba $t$ de student para comparar medias Se utilizó la prueba $t$ de student cuya fórmula sirve para establecer diferencias que se ha producido en el grupo experimental, a través de ello se estableció el nivel de retención en la memoria musical de los estudiantes del I semestre del curso de lenguaje musical. 


$$
t=\frac{\bar{Y}_{1}-\bar{Y}_{2}}{\sqrt{\frac{S C_{1}+S C_{2}}{n_{1}+n_{2}-2}\left(\frac{1}{n_{1}}+\frac{1}{n_{2}}\right)}}
$$

\section{Donde:}

$t \quad=$ t Calculada

$\mathrm{Y}_{1} \mathrm{Y}_{2}=$ Media aritmética

$\mathrm{SC}_{1} \mathrm{SC}_{2}=$ Varianza

$\mathrm{n}_{1} \mathrm{n}_{2}=$ Muestra

\section{RESULTADOS Y DISCUSIÓN}

La prueba pretest consto de tres partes, el porcentaje asignado fue en relación al peso de las interrogantes. La primera parte estuvo conformada por secuencias rítmicas, (comprensión) número de figuras, combinaciones y diseños rítmicos, se le asignó un $40 \%$. La segunda parte corresponde a las secuencias entonadas (reproducción) altura de los sonidos en el pentagrama y asimilación del sonido, se le asignó un $30 \%$. La tercera parte corresponde a la secuencia hablada (asimilación) reconocimiento de las notas en el pentagrama, se le asignó un 30\%, luego de tabular los resultados de los dos grupos, se obtuvo lo siguiente:

Tabla 6.

Promedio de la Prueba Pretest GE

\begin{tabular}{|c|c|c|c|c|c|c|c|c|}
\hline \multirow{3}{*}{$\mathrm{N}^{\circ}$ Est. } & \multicolumn{8}{|c|}{ G. Experimental Pretest } \\
\hline & \multicolumn{2}{|c|}{$\mathrm{O} 1$} & \multicolumn{2}{|c|}{$\mathrm{O} 2$} & \multicolumn{2}{|l|}{$\mathrm{O} 3$} & \multicolumn{2}{|c|}{ Total } \\
\hline & fi & $\%$ & $\mathrm{fi}$ & $\%$ & fi & $\%$ & $\mathrm{fi}$ & $\%$ \\
\hline 1 & 9 & $47 \%$ & 7 & $33 \%$ & 11 & $55 \%$ & 9 & $45 \%$ \\
\hline 2 & 9 & $47 \%$ & 11 & $53 \%$ & 14 & $68 \%$ & 11 & $56 \%$ \\
\hline 3 & 11 & $53 \%$ & 8 & $38 \%$ & 10 & $50 \%$ & 9 & $47 \%$ \\
\hline 4 & 11 & $57 \%$ & 4 & $18 \%$ & 19 & $93 \%$ & 11 & $56 \%$ \\
\hline 5 & 14 & $70 \%$ & 4 & $20 \%$ & 14 & $70 \%$ & 11 & $53 \%$ \\
\hline 6 & 9 & $47 \%$ & 9 & $45 \%$ & 3 & $15 \%$ & 7 & $36 \%$ \\
\hline 7 & 8 & $38 \%$ & 8 & $38 \%$ & 14 & $70 \%$ & 10 & $49 \%$ \\
\hline 8 & 7 & $33 \%$ & 4 & $20 \%$ & 10 & $50 \%$ & 7 & $34 \%$ \\
\hline 9 & 10 & $48 \%$ & 7 & $33 \%$ & 19 & $95 \%$ & 12 & $59 \%$ \\
\hline 10 & 14 & $68 \%$ & 9 & $45 \%$ & 10 & $48 \%$ & 11 & $54 \%$ \\
\hline 11 & 16 & $82 \%$ & 13 & $63 \%$ & 10 & $50 \%$ & 13 & $65 \%$ \\
\hline 12 & 11 & $53 \%$ & 15 & $73 \%$ & 8 & $40 \%$ & 11 & $55 \%$ \\
\hline 13 & 6 & $28 \%$ & 9 & $45 \%$ & 2 & $8 \%$ & 5 & $27 \%$ \\
\hline 14 & 15 & $75 \%$ & 19 & $93 \%$ & 14 & $70 \%$ & 16 & $79 \%$ \\
\hline 15 & 6 & $28 \%$ & 10 & $48 \%$ & 10 & $50 \%$ & 8 & $42 \%$ \\
\hline 16 & 16 & $78 \%$ & 9 & $45 \%$ & 5 & $23 \%$ & 10 & $49 \%$ \\
\hline 17 & 4 & $20 \%$ & 7 & $33 \%$ & 12 & $60 \%$ & 8 & $38 \%$ \\
\hline 18 & 12 & $58 \%$ & 4 & $20 \%$ & 13 & $63 \%$ & 9 & $47 \%$ \\
\hline 19 & 13 & $65 \%$ & 14 & $70 \%$ & 11 & $55 \%$ & 13 & $63 \%$ \\
\hline 20 & 3 & $13 \%$ & 18 & $88 \%$ & 7 & $35 \%$ & 9 & $45 \%$ \\
\hline PR & 10 & $50 \%$ & 0 & 460 & 11 & & & $50 \%$ \\
\hline
\end{tabular}

En la tabla 6, se presentan los resultados de la aplicación de la prueba pretest de los tres objetivos planteados del grupo de trabajo experimental, donde podemos observar que el grupo ha podido alcanzar un promedio de 10 de 20 puntos, que equivale a un $50 \%$.

El nivel de retención de la memoria musical del grupo experimental está en inicio, ya que para llegar a las competencias del curso de Lenguaje Musical se debe tener mayor desarrollo de esta; dicha prueba se corrobora con el análisis de los diarios de campo, donde observamos que los estudiantes no tienen un desarrollo pleno de la memoria musical. Por otro lado, podemos afirmar que los estudiantes si logran resolver algunos ejercicios simples. En el Grupo experimental el puntaje más alto que se logró obtener en la prueba pretest, está en la ubicación $\mathrm{n}^{\circ} 14$ con 16 de 20 puntos equivalente a $79 \%$, esto significa que el estudiante logró responder 15 de 20 preguntas, y el puntaje más bajo que se tiene es del estudiante en la ubicación $\mathrm{n}^{\circ} 13$, con 05 de 20 puntos equivalente a $27 \%$, el cual significa que solo logro responder 2 de 20 preguntas. Resultados que se corroboran con Berrón, Balsera, y Monreal (2016), un trabajo consciente de la atención y la capacidad de retención en la memoria aporta a los alumnos en su aprendizaje.

Tabla 7.

Promedio de la Prueba Pretest GC

\begin{tabular}{lllllllll}
\hline \multicolumn{7}{c}{ G. Control Pretest } \\
N ${ }^{\circ}$ Est. & O1 & O2 & O3 & Total \\
& fi $\%$ & fi & $\%$ & fi & $\%$ & fi & $\%$ \\
\hline 1 & 7 & $35 \%$ & 16 & $74 \%$ & 10 & $48 \%$ & 11 & $52 \%$ \\
2 & 7 & $35 \%$ & 9 & $43 \%$ & 7 & $33 \%$ & 8 & $37 \%$ \\
3 & 16 & $78 \%$ & 4 & $19 \%$ & 10 & $48 \%$ & 10 & $48 \%$ \\
4 & 5 & $25 \%$ & 18 & $86 \%$ & 14 & $64 \%$ & 12 & $59 \%$ \\
5 & 8 & $40 \%$ & 12 & $57 \%$ & 11 & $50 \%$ & 10 & $49 \%$ \\
6 & 13 & $63 \%$ & 6 & $26 \%$ & 6 & $26 \%$ & 8 & $39 \%$ \\
7 & 15 & $71 \%$ & 4 & $19 \%$ & 13 & $62 \%$ & 11 & $51 \%$ \\
8 & 14 & $67 \%$ & 6 & $26 \%$ & 13 & $62 \%$ & 11 & $52 \%$ \\
9 & 7 & $35 \%$ & 9 & $40 \%$ & 10 & $48 \%$ & 9 & $41 \%$ \\
10 & 14 & $65 \%$ & 7 & $31 \%$ & 8 & $38 \%$ & 9 & $45 \%$ \\
11 & 8 & $40 \%$ & 8 & $38 \%$ & 13 & $60 \%$ & 10 & $46 \%$ \\
12 & 7 & $35 \%$ & 8 & $38 \%$ & 20 & $93 \%$ & 12 & $55 \%$ \\
13 & 8 & $40 \%$ & 13 & $60 \%$ & 9 & $40 \%$ & 10 & $47 \%$ \\
14 & 5 & $22 \%$ & 12 & $57 \%$ & 13 & $60 \%$ & 10 & $46 \%$ \\
15 & 8 & $38 \%$ & 12 & $57 \%$ & 12 & $57 \%$ & 11 & $51 \%$ \\
16 & 10 & $46 \%$ & 13 & $62 \%$ & 17 & $81 \%$ & 13 & $63 \%$ \\
17 & 16 & $75 \%$ & 7 & $33 \%$ & 8 & $36 \%$ & 10 & $48 \%$ \\
18 & 3 & $14 \%$ & 10 & $45 \%$ & 8 & $38 \%$ & 7 & $33 \%$ \\
19 & 7 & $35 \%$ & 12 & $57 \%$ & 10 & $45 \%$ & 10 & $46 \%$ \\
20 & 9 & $43 \%$ & 7 & $33 \%$ & 8 & $36 \%$ & 8 & $37 \%$ \\
21 & 6 & $27 \%$ & 11 & $52 \%$ & 15 & $69 \%$ & 10 & $50 \%$ \\
PROMEDIO & 9 & $44 \%$ & 10 & $45 \%$ & 11 & $52 \%$ & 10 & $47 \%$ \\
\hline Fuente: & Resultados de la prueba & Pretest & GC & & & \\
\hline
\end{tabular}


En la tabla 7, se presentan los resultados de la aplicación de la prueba pretest de los tres objetivos planteados del grupo de trabajo control, donde podemos observar que el grupo ha podido alcanzar un promedio de 10 de 20 puntos, que equivale a un $47 \%$.

El nivel de retención de la memoria musical del grupo control está en inicio, ya que para llegar a las competencias del curso se debe tener mayor desarrollo de esta, dicha prueba se corrobora con el análisis de los diarios de campo, donde observamos que los estudiantes no tienen un desarrollo pleno de la memoria musical. Por otro lado, podemos afirmar que los estudiantes si logran resolver algunos ejercicios simples. En el Grupo control el puntaje más alto que se logró obtener en la prueba pretest, es del estudiante que se encuentran en la ubicación $n^{\circ} 16$ con 13 de 20 puntos equivalente a $63 \%$, esto significa que el estudiante logró responder 11 de 20 preguntas, y el puntaje más bajo se tiene en la ubicación $\mathrm{n}^{\circ} 18$, con 7 de 20 puntos equivalente a $33 \%$, el cual significa que solo logro responder 5 de 20 preguntas. Resultados que se corroboran con Garcia (2016), que indica estudiantes y docentes deben de conocer del estudio de la memoria musical, de no conocer ello siempre se optendran resultados bajos en el aprendizaje de la música en general.

La prueba postest constó de tres partes a las cuales el porcentaje asignado fue en relación al peso de las interrogantes. La primera parte de secuencias rítmicas (figuras, combinaciones y diseños rítmicos), se le asignó un 40\%. La segunda parte corresponde a las secuencias entonadas (entonación de las notas y melodías cortas), se le asignó un $30 \%$. La tercera parte corresponde a las secuencias habladas (reconocimiento de las notas en el pentagrama), se le asignó un 30\%. Luego de tabular los resultados de los tres grupos, se obtuvo lo siguiente:
Tabla 8.

Promedio de la Prueba Postest GE

\begin{tabular}{|c|c|c|c|}
\hline \multirow{3}{*}{$\mathrm{N}^{\circ}$} & \multicolumn{3}{|c|}{ G. Experimental Postest } \\
\hline & $\mathrm{O} 1$ & $\mathrm{O} 3$ & Total \\
\hline & fi $\%$ & fi $\%$ & fi $\%$ \\
\hline 1 & $1680 \% 1890 \%$ & $1888 \%$ & $1786 \%$ \\
\hline 2 & $1785 \% 1888 \%$ & $1888 \%$ & $1787 \%$ \\
\hline 3 & $1783 \% 2098 \%$ & $1993 \%$ & $1891 \%$ \\
\hline 4 & $1577 \% 1993 \%$ & $1993 \%$ & $1787 \%$ \\
\hline 5 & $1785 \% 1995 \%$ & $1993 \%$ & $1891 \%$ \\
\hline 6 & $1995 \% 2098 \%$ & $1888 \%$ & $1994 \%$ \\
\hline 7 & $1892 \% 1993 \%$ & $1995 \%$ & $1993 \%$ \\
\hline 8 & $1888 \% 1888 \%$ & $1995 \%$ & $1890 \%$ \\
\hline 9 & $1890 \% 1890 \%$ & $20100 \%$ & $1993 \%$ \\
\hline 10 & $1993 \% 1888 \%$ & $1995 \%$ & $1892 \%$ \\
\hline 11 & $1783 \% 1993 \%$ & $1890 \%$ & $1889 \%$ \\
\hline 12 & $1787 \% 1888 \%$ & $1993 \%$ & $1889 \%$ \\
\hline 13 & $1993 \% 1888 \%$ & $1783 \%$ & $1888 \%$ \\
\hline 14 & $1993 \% 1890 \%$ & $1993 \%$ & $1892 \%$ \\
\hline 15 & $1682 \% 1888 \%$ & $1993 \%$ & $1787 \%$ \\
\hline 16 & $1995 \% 1993 \%$ & $1785 \%$ & $1891 \%$ \\
\hline 17 & $1783 \% 1888 \%$ & $1890 \%$ & $1787 \%$ \\
\hline 18 & $1785 \% 1993 \%$ & $1993 \%$ & $1890 \%$ \\
\hline 19 & $1892 \% 1995 \%$ & $1785 \%$ & $1891 \%$ \\
\hline 20 & $1783 \% 1995 \%$ & $1888 \%$ & $1889 \%$ \\
\hline PROMEDIO & $1787 \% 1891 \%$ & $1891 \%$ & $1890 \%$ \\
\hline
\end{tabular}

En la tabla 8, se presentan los resultados de la aplicación de la prueba postest de los tres objetivos planteados del grupo de trabajo experimental, donde podemos observar que el grupo ha podido alcanzar un promedio de 18 de 20 puntos, que equivale a un $90 \%$.

El nivel de retención de la memoria musical del grupo experimental está en logro esperado, demostrando que ya cuentan con las competencias del curso, a su vez la prueba se corrobora con el análisis de los diarios de campo. Por otro lado, los estudiantes en su mayoría logran resolver los ejercicios simples mediante la memoria musical. En el grupo experimental el puntaje más alto que se logró obtener se encuentra en la ubicación 
$\mathrm{n}^{\circ} 6$ con 19 de 20 puntos equivalente a $94 \%$, esto significa que el estudiante logró responder 18 de 20 preguntas, y el puntaje más bajo que se tiene es del estudiante en la ubicación $\mathrm{n}^{\circ} 1$, con 17 de 20 puntos equivalente a $86 \%$, el cual significa que logro responder 16 de 20 preguntas. Resultados que concuerdan con Balo (2015), donde indica que para realizar correctamente los diferentes tipos de dictados, se requiere el desarrollo de dos capacidades: memorizar lo escuchado y escribirlo en un papel utilizando los códigos propios de la lectoescritura.

Tabla 9.

Promedio de la Prueba Postest GC

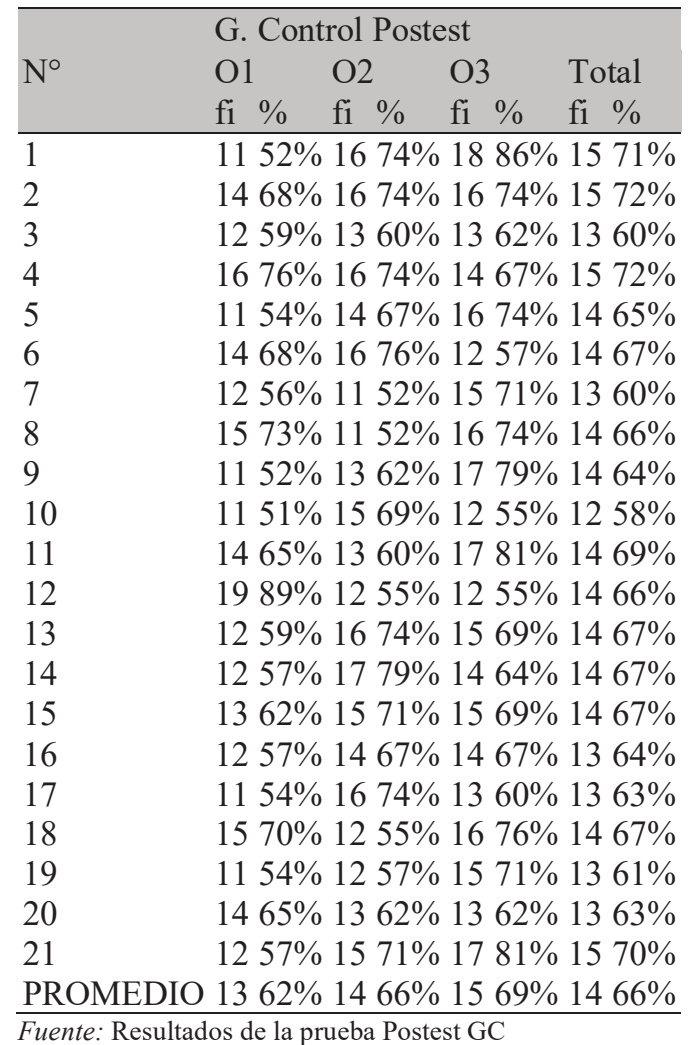

En la tabla 9, se presentan los resultados de la aplicación de la prueba postest de los tres objetivos planteados del grupo de trabajo control, donde podemos observar que el grupo ha podido alcanzar un promedio de 14 de 20 puntos, que equivale a un $66 \%$.

Se puede manifestar que el nivel de retención de la memoria musical del grupo control está en logro previsto, ya que cuentan con un mayor desarrollo para llegar a las competencias del curso, dicha prueba se corrobora con el análisis de los diarios de campo. Por otro lado, podemos afirmar que los estudiantes en su mayoría logran resolver los ejercicios simples. En el grupo control el puntaje más alto que se logró, es del estudiante en la ubicación $\mathrm{n}^{\circ} 2$ con 15 de 20 puntos equivalente a $72 \%$, esto significa que el estudiante logró responder 15 de 20 preguntas, y el puntaje más bajo que se tiene es del estudiante en la ubicación $\mathrm{n}^{\circ} 10$, con 12 de 20 puntos equivalente a $58 \%$, el cual significa que logro responder 11 de 20 preguntas.

Los Resultados Obtenidos se asemejan a los estudios realizados por Martinez (2008), donde concluye que el desarrollo de la competencia auditiva, educación o formación del oído musical a través de la memoria, es un proceso lento pero firme, por lo tanto exitoso, cuando se trabaja simultáneamente la lectura, escritura, audición y ejecución musical a través del canto o de la interpretación de un instrumento musical.

Otro estudio de Velásquez (2010), concluye que la memoria musical es un elemento indispensable en la formación de un músico, ya que permite la individualización de los elementos de la música en el cerebro logrando una mejor asimilación de todos los elementos musicales.

Tabla 10.

Estadísticas de muestras emparejadas

\begin{tabular}{lll} 
Media & N $\begin{array}{l}\text { Desviación } \\
\text { estándar }\end{array}$ & $\begin{array}{l}\text { Media } \\
\text { de error } \\
\text { estándar }\end{array}$ \\
Par exp_pretest_fi $10,0000202,42899$ &, 53005 \\
$1 \quad$ exp_postest_fi 17,9048 20,62488 &, 13636 \\
\hline Fuente: Prueba Estadística.
\end{tabular}

Tabla 11.

Correlación de muestras emparejadas

\begin{tabular}{lllr} 
& & \multicolumn{2}{c}{ N Correlación Sig. } \\
$\begin{array}{l}\text { Par } \\
1\end{array} \quad$ exp_pretest_fi \& & 20,066 &, 777 \\
\hline Fuente: Prueba Estadística. & &
\end{tabular}

En la tabla 10 y 11 se describen las mediciones a comparar y se presenta la correlación entre las mismas del grupo experimental, donde observamos que la media para el pretest es de 10,00 y para el postest es de 17,90, la desviación estándar es para el pretest de 2,42 y para el postest es de 0,62 , donde la media de error para el pretest es de 0,53 y para el postest es de 0,13 y el grado de correlación es de 0,66 con un nivel de significancia de 0,77 . Lo que indica que si existe un nivel de significancia alta. 
Tabla 12.

Prueba de muestras emparejadas

\begin{tabular}{|c|c|c|c|c|c|c|c|c|}
\hline & \multicolumn{5}{|c|}{ Diferencias emparejadas } & $\mathrm{t}$ & $\mathrm{gl}$ & $\begin{array}{c}\text { Sig. } \\
\text { (bilateral) }\end{array}$ \\
\hline & \multirow[t]{2}{*}{ Media } & \multirow[t]{2}{*}{$\begin{array}{c}\text { Desviación } \\
\text { estándar }\end{array}$} & \multirow{2}{*}{$\begin{array}{c}\text { Media de } \\
\text { error } \\
\text { estándar }\end{array}$} & $\begin{array}{r}95 \% \text { de in } \\
\text { confian } \\
\text { difer }\end{array}$ & $\begin{array}{l}\text { itervalo de } \\
\text { za de la } \\
\text { encia }\end{array}$ & & & \\
\hline & & & & Inferior & Superior & & & \\
\hline Par $1 \begin{array}{l}\text { exp_pretest_fi - } \\
\text { exp_postest_fi }\end{array}$ & $-7,90476$ & 2,46789 &, 53854 & $-9,02813$ & $-6,78139$ & $-14,678$ & 19 &, 000 \\
\hline
\end{tabular}

Fuente: Prueba Estadística.

En la tabla 12 se presenta a la prueba estadística propiamente dicha en la que se describen la diferencia de la media, la desviación estándar de las diferencias, el error estándar de diferencias y finalmente la prueba $t$. del grupo experimental en la aplicación del pre y postest y se afirma: que si encontró diferencias en el nivel de retención de la memoria musical de diferentes secuencias, entre la medición del inicio (pretest) y la medición hecha al finalizar la intervención (postest), ya que se observa un valor de $t$ de $-14,6 g l=19$ grado de libertad y $p=0,000$, menor que 0,05 por lo que el nivel de retención de la memoria musical de diferentes secuencias, es diferente entre la primera (pretest) y la segunda (postest) medición; por lo tanto se rechaza la hipótesis $\mathrm{H}_{\mathrm{o}} \mathrm{y}$ se acepta la hipótesis $\mathrm{H}_{\mathrm{a}}$.

\section{Validación de la hipótesis general}

De los datos obtenidos de 2,09>1,72; es decir que: $\mathrm{X}_{\mathrm{e}}>\mathrm{X}_{\mathrm{c}}$ se acepta la hipótesis alterna y se rechaza la hipótesis nula, luego se concluye que el nivel de retención en la memoria musical en el grupo experimental y de control es diferente después del tratamiento experimental.

\section{CONCLUSIONES}

El nivel de retención en la memoria musical de diferentes secuencias rítmicas, entonadas y habladas de notas musicales, según la aplicación de tres métodos: Dalcroze, LenMus, y la Relación Sonido Color ha mejorado significativamente, ya que la mayoría de estudiantes $(80 \%)$ en la prueba postest se ubicaron en la escala de Logro Esperado, en comparación a la prueba pretest, donde la mayoría de estudiantes $(45 \%)$ se ubicaron en la escala En Inicio, los estudiantes intervenidos consiguieron el desarrollo de su memoria musical con ayuda de los métodos empleados, logrando resolver las diferentes combinaciones de dictados tanto rítmicos, melódicos y de reconocimiento de las notas en el pentagrama, superando así el nivel de retención en la memoria musical.

El nivel de retención en la memoria musical de secuencias rítmicas, según la aplicación del método Dalcroze, es significativo en un nivel de Logro Previsto, ya que la mayoría de estudiantes $(45 \%)$ lograron interiorizar el ritmo a través del movimiento, desarrollar el oído a través de la escucha de diversas combinaciones de rítmicas, dominar las combinaciones de los principales patrones rítmicos entre blancas y negra, negra y corchea para asimilarlas y luego reproducirlas, en comparación de la prueba pretest que la mayoría de estudiantes (55\%) estaban en un nivel De Inicio de su aprendizaje.

El nivel de retención en la memoria musical de secuencias entonadas, según la aplicación del método LenMus, es significativo en un nivel de Logro Esperado, ya que los estudiantes en su totalidad $(100 \%)$ lograron solfear con la entonación adecuada, reconocer los intervalos de segunda y tercera mayor y menor cuarta justa, en comparación de la prueba pretest que la mayoría de estudiantes $(75 \%)$ se encontraban en un nivel de Inicio de su aprendizaje.

El nivel de retención en la memoria musical de secuencias habladas, según la aplicación del método Relación Sonido Color, es significativo en un nivel de Logro Esperado, ya que la mayoría de estudiantes (85\%) lograron reconocer las notas musicales y su dinámica en el pentagrama mediante los colores, para su asimilación y su reproducción, en comparación de la prueba pretest donde los estudiantes en su mayoría (50\%) estaban en inicio de su aprendizaje. 


\section{Agradecimiento}

Nuestro agradecimiento a los estudiantes del Programa de Música del I semestre 2018 II de la Escuela Profesional de Arte, Universidad Nacional del Altiplano por permitirnos realizar la investigación.

\section{Conflictos de interés}

El grupo de investigadores no tiene ningún conflicto de intereses.

\section{REFERENCIAS BIBLIOGRÁFICAS}

Alvarado, R. (2013). La Música y su Rol en la Formación del Ser Humano. Santigo: Universidad de Chile. Recuperado de http://repositorio.uchile.cl/bitstream/ handle/2250/122098/La musica_y su rol en la formacion del ser humano. pdf; sequence $=1$

Aschero, S. (2012). Numerofonia. Recueprado de Aschero Opus: https://sergioaschero.com. ar/descarga/numerofonia/Teoria $\% 20 \mathrm{de} \% 20$ la $\% 20$ Numerofonia $\% 20 \mathrm{de} \% 20$ Aschero.pdf

Balo, M. (2015). La diversidad tímbrica en el dictado musical a dos voces como estrategia para superar las dificultades de transcripción. Revista Electronica LEEME (36), 1-16.

Barbacci, R. (1965). Educación de la Memoria Musical. Buenos Aires: Ricordi Americana Editorial.

Barraza, A. (2006). Apuntes Sobre Metodología de la Investigación. Investigación Educativa, 5-17.

Berrón, E., Balsera, F. y Monreal, I. (2016). Desarrollo de la Memoria en la Asignatura de Lenguaje Musical. Lista Electronica Europea de Música en la Educación LEEME (38), 1735.

Bowers, B. (2003). Brain Images Reveal Cerebral Side of Music. EE.UU: Science News.

Campbell, D. y Stanley, J. (1996). Experiemtnal and quasi-Experimental designs for reserarch. Chicago: Rand Mcnally.

Cerda, H. (1993). Los Elementos de la Invetigación. Quito, Ecuador: El Buho LTDA.

Chaffin, R. y Imreh, G. (2002). Practicing Perfection: Piano Performance as expert memory. Psychological Science, 13(4), 342349.

Chaffin, R. y Logan, T. (2006). Practicing perfection: how concert soloists prepare for performance. Advances in Cognitive Psychology, 2 (2-3), 113-130.

Cidoncha, P. (2011). Colormúsica. Recuperado de www.colormusica.com: http://www. colormusica.com/recursos/recursos.html

Clynes, M. (1982). La Música, la Mente y el Cerebro. New York.

Cohen, M. y Manion, L. (2003). Métodos de Invetigación Educativa. Madrid, España: Muralla.

Córdoba, D., Albert, J. y López, S. (16 de setiembre de 2010). Potenciación a largo plazo en la corteza humana. doi: https://doi. org/10.33588/rn.5106.2009616

Cromie, W. J. (1997). How your Brain Listens to Music. New York: Harvard Gazette Archives.

Echeverría, R. (2002). Ontología del Lenguaje. España: Dolmen Ediciones Océano.

Feldman, R. (2005). Psicología con Aplicaciones en países de habla hispana. México: McGraw Hill.

Garcia, J. (2016). La Memoria Musical en los Estudiantes de Piano: Visión y Propuesta. Revista de Educación y Humanidades DEDiCA (10), 13-27.

Gardner, H. (1994). Estructura de la Mente. La teoria de las Inteligencias Multiples. Mexico: F.C.E. $2^{\circ}$ edicción.

Hernández, R., Fernandez, C. y Baptista, P. (2014). Metodología de la Invetigación (6ta edición ed.) Mexico: McGraw Hill.

Herrera, M. y Cremades, R. (2012). Estudio Descriptivo Sobre el Uso de la Memoria Musical en Estudiantes de Piano del Estado de Chihuahua, México. Revista de Educación y Humanidades, 2(2), 279-294.

Ibarra, R. (2009). Neuroanatomía y Neurofisiología del Aprendizaje y Memoria Musical. Boletin elcetrónico de Investigación de la Asociación Oaxaqueña de Psicologia A.C., 5(1), 39-51.

Jaques, D. G. (1999). Institute Jaques-Dalcroze Genéve. Recuperado de http://www.dalcrozc. $\mathrm{ch} /$

Martinez, F. (2008). Incidencia de la Memoria Musical en el Desarrollo de la Competencia Auditiva. (Tesis de Maestria, Universidad Pedadgógica Nacional de Bogota) Recueprado de http://www.laguitarra-blog. com/wp-content/uploads/2012/01/incidenciamemoria-musical-competencia-auditiva.pdf

Noice, H., Jefferey, J., Noice, T. y Chaffin, R. (2008). Memorization by a jazz musician: acase study. Psychology of Music, 36(1), 6379. 
Pino, F. (2011). vix.com. Recuperado el 15 de 01 de 2019 de https://www.vix.com/es/btg/ curiosidades/2011/01/15/que-es-la-memoriahumana

Ráez, J. (2013). La Rítmica de Dalcroze. Recuperado el 22 de 12 de 2019, de https:// www.slideshare.net/leymycastillovargas/ dalcroze-28940243

Riera, N. (2011). Relación Sonido-Color en la Experiencia Sinestésica de la Música Clásica. (tesis de Licenciatura, Universidad Centroccidental Lisandro Alvarado). Recuperado de https://eternobisiesto.files. wordpress.com $/ 2013 / 07 /$ relacic3b3n-colorsonido.pdf

Rodriguez, G. (1996). Metodología de la Investigación Cualitativa. Malga: Aljibe.

Rodriguez, S. J. (2005). La Investigación Acción Educativa ¿qué es? ¿cómo se hace? Lima: ali arte gráfico publicaciones srl.

Salmerón, C. (2013). LenMus Software abierto para la Teoría Musical. Recuperado el 21 de 01 del 2019 de LenMus Software abierto para la Teoría Musical: http://www.lenmus.org/en/ phonascus/intro

Sanches, J., Flores, H. y Aravena, G. (2003). Desarrollo de la memoria de niños con discapacidad visual a través del audio. Santiago: Universidad de Chile.

Sandoval, C. (2015). Evaluación del impacto de Actividades de Aprendizaje Musicales en el Desarrollo de la Memoria Operativa en Niños de Nivel de Transición 2. (Tesis de Maestria, Pontificia Universidad Católica de Chile) Recuperado de https://repositorio. uc.cl/bitstream/handle/11534/15772/664421. pdf? sequence $=1$.

Shinn, F. (1984). Musical Memory and its Cultivaion. London: Geber Press.

Sloboda, J. H. (1985). An exceptional musical memory. Music perception. EE.UU.

Velásquez, Y. H. (2010). Propuesta de ejercicios para el desarrollo de la memoria. (Tesis de Licenciatura, Universidad Pedagógica Nacional de Bogota). Recuperado de http:// www.laguitarra-blog.com/wp-content/ uploads/2012/08/propuestas-de-ejerciciospara-el-desarrollo-de-la-memoria-de-losguitarristas.pdf

Weinberg, N. M. (2006). Musica y el Cerebro. Scientific American.

Willems, E. (2001). El oído musical. La preparación auditiva del niño. Barcelona España: Paidós Educador. 